\title{
Development of a variance prioritized multiresponse robust design framework for quality improvement
}

\author{
Jami Kovach \\ Department of Information and Logistics Technology, University of Houston, \\ Houston, Texas 77204, USA \\ Byung Rae Cho \\ Advanced Quality Engineering Laboratory, Department of Industrial Engineering, \\ Clemson University, Clemson, South Carolina 29634, USA

\begin{abstract}
Jiju Antony
Strathclyde Institute of Operations Management, Design Manufacturing and Engineering Management, University of Strathclyde, Glasgow, G1 1XY, UK
\end{abstract}

Submitted to:

International Journal of Quality and Reliability Management

February 2008

* Corresponding author 


\title{
Development of a variance prioritized multiresponse robust design framework for quality improvement
}

\author{
Jami Kovach \\ Department of Information and Logistics Technology, University of Houston, \\ Houston, Texas 77204, USA \\ Byung Rae Cho \\ Advanced Quality Engineering Laboratory, Department of Industrial Engineering, \\ Clemson University, Clemson, South Carolina 29634, USA \\ Jiju Antony \\ Strathclyde Institute of Operations Management, Design Manufacturing and Engineering \\ Management, University of Strathclyde, Glasgow, G1 1XY, UK
}

\begin{abstract}
Purpose - Robust design is a well-known quality improvement method that focuses on building quality into the design of products and services. Yet, most well established robust design models only consider a single performance measure and their prioritization schemes do not always address the inherent goal of robust design. In this paper, we propose a new robust design method for multiple quality characteristics where the goal is to first reduce the variability of the system under investigation and then attempt to locate the mean at the desired target value.
\end{abstract}

Design/methodology/approach - In this paper, we investigate the use of a response surface approach and a sequential optimization strategy to create a flexible and structured method for modeling multiresponse problems in the context of robust design. Nonlinear programming is used as an optimization tool.

Findings - Our proposed methodology is demonstrated through a numerical example and the results are compared to that of the traditional robust design method. The proposed methodology provides enhanced optimal robust design solutions consistently.

Originality/value - This paper is perhaps the first study on the prioritized response robust design with the consideration of multiple quality characteristics. The findings and key observations of this paper will be of significant values to the quality and reliability engineering/management community.

Keywords Quality, robust design, multiresponse problems

Paper type Reserch paper 


\section{Introduction}

In today's industrial production environment, it is essential that companies create and retain a competitive advantage over their competitors with respect to quality in order to be successful. The robust design (RD) methodology, first developed by Taguchi $(1986,1987)$, is important to industrial quality improvement initiatives. This approach focuses on building quality into the design of products and services though the determination of the optimum operating conditions in order to minimize performance variability and deviation from the target value of interest (i.e. process bias). Since it was first introduced, this approach has come under serious criticism due to the statistical analysis methods and optimization approaches utilized. In his method of RD, Taguchi advocates minimizing signal-to-noise ratios to determine the best overall combination of design parameter settings and identifying adjustment factors, which are used to adjust the mean to the desired target value. Yet, Nair and Shoemaker (1990) argue that by simply collapsing experimental data into signal-to-noise ratios much of the information concerning the system's behavior is lost. Additionally, Taguchi gives no real justification for the use of these ratios, and the details surrounding the use of adjustment factors to achieve the target value of interest are sketchy at best. To address these issues, there have been several attempts in the literature to improve the analysis and optimization phases of the RD methodology.

Several RD optimization models that are relevant to the work presented here are based on the dual response approach, which was first considered by Myers and Carter (1973). Here, the process mean and variance of a single quality characteristic are modeled separately using response surfaces. These functions are then optimized simultaneously to determine the system's optimum operating parameters. The first attempt to combine this type of optimization model within the RD methodology was developed by Vining and Myers (1990). As an extension to this 
dual response approach to $\mathrm{RD}$, Lin and $\mathrm{Tu}$ (1995) proposed the mean-squared error (MSE) model. This approach relaxed the zero-bias assumption of the previous model to provide solutions that are better (or at least equal), in terms of the variance achieved, through a more flexible optimization model. Lin and Tu (1995) further suggested assigning different weights to process bias and variability as a way of prioritizing the optimization procedure. Along these lines, Park and Cho (2003), Shin and Cho (2005), and Cho and Park (2005) considered more complex RD systems from the viewpoints of non-normal data, process oriented modeling, and unbalanced design space, respectively, Finally, Kovach and Cho (2006) and Lee et al. (2007) developed RD models for unbalanced data structures and irregular experimental situations, respectively.

The RD optimization models discussed thus far may be considered multiresponse approaches because they simultaneously optimize the response models for both the process mean and variance. These approaches, however, only consider the response models for the process mean and variance for a single performance measure. Yet, customer judge products on multiple scales simultaneously. Further, the prioritization scheme of these optimization models do not always address the inherent goal of RD. Taguchi $(1986,1987)$ believed it was vital to quality improvement efforts to not simply focus on adjusting the process mean to the desired target value. He felt strongly that it was also critical to reduce the variability of the process around the mean. Therefore, when optimizing a system, RD should consider multiple quality characteristics simultaneously and place top priority on minimizing process variability. In this paper, we propose a new approach to the multiresponse RD problem to address these issues, called variance-prioritized multiresponse robust design (VPMRD). In this approach, the optimization phase of $\mathrm{RD}$ is formulated as a nonlinear goal programming problem where the prioritization 
scheme is specified using a preemptive optimization procedure. Through a numerical example, we demonstrate the use of this methodology to determine the optimum operating conditions for the system under investigation based on experimentation and system modeling.

In the next section, we describe our proposed methodology in detail. Then, an example is used to demonstrate the practical implementation of our proposed approach. Next, a comparison study is conducted to validate the proposed model. Finally, we discuss conclusions concerning the practical implications of the proposed approach.

\section{The proposed methodology}

While there are several optimization models that can be employed within the RD methodology, few consider the inherent goal of RD. Recall that Taguchi's quality philosophy suggests that quality improvement efforts should not simply focus on the mean; it also involves the variability around the mean. Yet, well-established optimization approaches, including the dual response (Vining and Myers, 1990) and MSE models (Lin and Tu, 1995), may have failed to place top priority on minimizing the variance. Therefore, we propose a new method of RD that creates a structured, yet flexible modeling approach for multiresponse RD problems where the goal is to first reduce the variability of the system under investigation and then attempt to reduce the process bias. In this new approach, we utilize nonlinear goal programming techniques [3] to determine the system's optimum operating conditions based on response surface models of the system derived though experimentation and analysis.

The methodology proposed in this paper is shown in Figure 1, which consists of first determining the problem to be considered and choosing the responses of interest, as well as the design factors, to be included in the investigation. This information is then used to create the 
appropriate experimental design, and the experiment is carried out according to plan. Next, data collected from the experiment are analyzed using the least squares method of regression analysis. Based on these results, response surface models for the mean and variance of each response are created. Finally, the proposed VPMRD model is used to simultaneously optimize the responses to obtain the settings for the design parameters that make the system under investigation perform optimally relative to minimizing the process' variance and bias.

[Figure 1 Approximately Here]

\section{Planning}

In an RD study, the determination of the quality characteristics of interest is strongly related to the nature of the problem being investigated. Similarly, the choice of experimental parameters is often derived from the responses under investigation using prior engineering knowledge concerning the system being studied. In many situations, however, practitioners may not have sufficient experience to effectively choose the appropriate experimental parameters. Therefore, the alternative is to collect data relative to potential experimental factors and then use multivariate studies, correlation analysis, and/or screening experiments to determine the specific factors to consider in an RD investigation.

\section{Experimentation}

In designing an experiment, there are many standard approaches from which to choose. These include, but are not limited to, factorial designs, Taguchi designs, and response surface designs. The key, therefore, is to choose the design that best addresses the experimental question and supports the desired data analysis given the available resources (i.e. time and experimental budget). The size of the experiment is determined in part by the number of factors included, the 
number of levels of each factor to be tested, and the number of replications planned. The results obtained form the experiment provides the information necessary to create response surface models that describe the behavior of the system under investigation.

\section{System modeling}

To create response surface models for the mean and variance of each quality characteristic under investigation, we utilize the least squares method of regression analysis. Using this approach, consider that each of the $n$ treatment combinations in an experiment consists of $r$ replicates. For each response of interest, let $y_{u j}$ represent the $j^{\text {th }}$ response at the $u^{\text {th }}$ treatment where $j=1,2, \ldots, r$ and $u=1,2, \ldots, n$. Then, for each quality characteristic, the mean and variance for the $u^{\text {th }}$ treatment can be estimated using the following equations:

$$
\bar{y}_{u}=\frac{\sum_{j=1}^{r} y_{u j}}{r} \text { and } s_{u}^{2}=\frac{\sum_{j=1}^{r}\left(y_{u j}-\bar{y}_{u}\right)^{2}}{r-1}
$$

for $u=1,2, \ldots, n$. Assuming the underlying distribution of the experimental data is normal with constant variance, the estimators given in Equation (1) are then used to create the response surface functions of the mean and variance based on the method of least squares regression analysis as follows. The model for the mean, therefore, is written as

$$
\hat{\mu}(\mathbf{x})=\mathbf{X} \hat{\boldsymbol{\beta}}
$$

where

$$
\hat{\boldsymbol{\beta}}=\left(\mathbf{X}^{\mathrm{T}} \mathbf{X}\right)^{-1} \mathbf{X}^{\mathrm{T}} \overline{\mathbf{y}}
$$


$\mathbf{X}$ is the design matrix, $\hat{\boldsymbol{\beta}}$ is the estimate of the vector of unknown model parameters, and $\overline{\mathbf{y}}$ is the vector of estimated means for each treatment combination in an experiment. In addition, the response surface function for the variance is given as

$$
\hat{\sigma}^{2}(\mathbf{x})=\mathbf{X} \hat{\gamma}
$$

where

$$
\hat{\gamma}=\left(\mathbf{X}^{\mathrm{T}} \mathbf{X}\right)^{-1} \mathbf{X}^{\mathrm{T}} \mathbf{s}^{2}
$$

$\hat{\gamma}$ is the estimate of the vector of unknown model parameters, and $\mathbf{s}^{2}$ is the vector of estimated variances for each treatment in an experiment. However, if the underlying distribution of the experimental data is skewed (i.e. is significantly non-normal) or the assumption of constant error-variance is violated significantly, the data will have to be transformed in order to proceed with this type of analysis. Commonly used transformations include, but are not limited to, a log transformation or square root transformation; the transformation to be used depends on the specific situation and the degree to which the necessary assumptions are violated. Assuming the necessary assumptions can be verified, the fitted response functions for each quality characteristic under investigation are then optimized simultaneously to determining the system's optimum operating parameters.

\section{Optimization}

The framework for the proposed optimization model is shown in Table 1 and is structured in such a way that given the system parameters and the models of their behavior, the goal is to find the design factor settings based on satisfying the system requirements and goals by minimizing the objective function. In our proposed model, $k$ responses of type $t$ are considered. 
These designations are necessary to accommodate the different types of quality characteristics that may be considered in an RD investigation. According to Taguchi, responses are usually categorized as one of the following three types.

1. Smaller-the-better (S-type): Minimize the quality characteristic of interest (i.e. the target value equals zero).

2. Nominal-the-better ( $N$-type): The quality characteristic of interest has a specific target value.

3. Larger-the-better (L-type): Maximize the quality characteristic of interest (i.e. the target value approaches infinity).

Further, $\hat{\mu}_{k t}(\mathbf{x})$ and $\hat{\sigma_{k t}^{2}}(\mathbf{x})$ are the response surface functions for the process mean and variance of response $y_{k t}$, respectively, which are assumed to be independent of each other, and $\tau_{\hat{\mu_{k t}}}$ and $\tau_{\hat{\sigma_{k t}^{2}}}$ are the desired target values for the mean and variance of response $y_{k t}$, respectively.

[Table 1 Approximately Here]

In the proposed model, the constraints consist of the system's technical requirements and its desired goals. The system requirements are characterized by the upper and/or lower limits on the system variables under consideration, which must be satisfied in order for the solution to be feasible. Further, the constraints representing the system goals model the deviation from the desired target values for both the mean and variance of each response. Overall, the system goal is to minimize the deviation from the desired target values. Additionally, we can specify the priority of individual goals in the objective function given this framework.

The objective function for the proposed model is formulated as a nonlinear goal programming problem (Hillier and Liberman, 2001). This type of approach makes the general multiresponse problem inherently easier to solve because the objective function can be modeled 
as a single function using deviation variables. In mathematical programming terms, deviation variables are also known as auxiliary variables. In the objective function for our proposed model, these variables are used to denote the under- and over-achievement of a constraint (i.e. a desired range or target value), which are given as $d_{g}^{-}$and $d_{g}^{+}$, respectively; hence, the deviation variables are also used in formulating the constraints for the proposed model. For example, consider the constraint associated with the process mean of a particular response of interest. Using deviation variables, this constraint can be written in general terms as

$$
d_{g}=\hat{\mu_{k t}}(\mathbf{x})-\tau_{\hat{\mu_{k t}}}, \text { for } g=1,2, \ldots, r
$$

where

$$
d_{g}=d_{g}^{+}-d_{g}^{-}
$$

and

$$
\begin{aligned}
& d_{g}^{+}=\left\{\begin{array}{cc}
d_{g} & \text { if } d_{g} \geq 0 \\
0 & \text { otherwise }
\end{array}\right. \\
& d_{g}^{-}=\left\{\begin{array}{cc}
\left|d_{g}\right| & \text { if } d_{g} \leq 0 \\
0 & \text { otherwise }
\end{array}\right.
\end{aligned}
$$

Given these definitions, the constraint in Equation (6) can then be rewritten as

$$
\hat{\mu_{k t}}(\mathbf{x})-\left(d_{g}^{+}-d_{g}^{-}\right)=\tau_{\hat{\mu_{k t}}} .
$$

Further, the method described here for modeling constraints using deviation variables for the mean of a quality characteristic can also be used for modeling the variance. Given this type of approach, the deviation variables associated with the constraints for the mean and variance of each response are then combined to form a single objective function as follows:

$\min Z=\left[f_{1}\left(d_{1}^{-}, d_{1}^{+}\right), f_{2}\left(d_{2}^{-}, d_{2}^{+}\right), \ldots, f_{r}\left(d_{r}^{-}, d_{r}^{+}\right)\right]$

s.t. $\quad$ Constraints due to system requirements: 
1. $S$-type quality characteristic: $\hat{\mu_{k S}}(\mathbf{x}) \leq \mathrm{USL}_{k S}$ for $k=1,2, \ldots, a$

2. $N$-type quality characteristic: $\mathrm{LSL}_{k N} \leq \hat{\mu_{k N}}(\mathbf{x}) \leq \mathrm{USL}_{k N}$ for $k=a+1, a+2, \ldots, b$

3. $L$-type quality characteristic: $\hat{\mu_{k L}}(\mathbf{x}) \geq \mathrm{LSL}_{k L}$ for $k=b+1, b+2, \ldots, c$

Constraints due to system goals:

1. Process mean:

$\hat{\mu_{k t}}(\mathbf{x})+d_{g}^{-}-d_{g}^{+}=\tau_{\hat{\mu_{k t}}}$ for $t=$ type of quality characteristic $(S-, N-$, or $L-)$

2. Process variance:

$$
\hat{\sigma_{k t}^{2}}(\mathbf{x})+d_{g}^{-}-d_{g}^{+}=\tau_{\hat{\sigma_{k t}^{2}}} \text { for } t=\text { type of quality characteristic }\left(S-, N-, \text { or } L_{-}\right)
$$

Bounds:

1. Design factors: $x_{i \min } \leq x_{i} \leq x_{i \max }$ for $i=1,2, \ldots, n$

2. Deviation variables: $d_{g}^{-}, d_{g}^{+} \geq 0$ and $d_{g}^{-} \cdot d_{g}^{+}=0$ for $g=1,2, \ldots, r$

In the proposed model, the constraints are delineated according to the type of quality characteristic considered where USL and LSL are the upper and lower specification limits, respectively, for the system's requirements. To establish a prioritization scheme for the optimization procedure, our proposed model utilizes a preemptive approach involving sequential optimization. Here, weights of different magnitudes are assigned to the deviation variables associated with the process mean and variance. In this model, we propose setting priorities in such a way that the goal is to first minimize the variance and then attempt to achieve the mean equal to the desired target value. The proposed approach is illustrated through the numerical example in the following section.

\section{Implementation of the proposed model}

Consider the problem of optimizing a chemical filtration process for measuring dosages with respect to filtration time, volume, and purity. Here, we consider temperature $\left(x_{1}\right)$ and pressure $\left(x_{2}\right)$ as design factors and filtration time $\left(y_{1 S}\right)$, filtration volume $\left(y_{2 N}\right)$, and filtration purity 
$\left(y_{3 L}\right)$ as our responses of interest. Hence, this becomes a multiresponse RD study, given that there are multiple quality characteristics of interest in determining the optimum operating conditions for the system under investigation. In this example, filtration time is considered an $S$ type quality characteristic since it is desirable to minimize processing time. Additionally, the system configuration has a maximum processing time of 7 seconds that cannot be exceeded. The desired target for the volume of the filtered chemical dose is $10 \mathrm{~mL}$, where the allowable tolerance is $\pm 0.5 \mathrm{~mL}$; therefore, filtration volume is considered an $N$-type response. Further, filtration purity is required to be as high as possible, therefore this response is considered an $L$ type quality characteristic with a natural bound at $100 \%$. In this particular example, it is critical that we reduce the variability of each response simultaneously in order to stabilize the processing cost in terms of filtration time and improve product quality by minimizing the occurrence of under- and over-filled vials and stabilizing filtration purity.

To estimate quadratic models of the responses, a central composite design with four center points is chosen for this experiment. Additionally, the experiment is replicated three times and data are collected concerning the responses of interest. The experimental design schemes and the data for each response are displayed in Tables 2-4, respectively.

[Table 2 - Approximately Here]

[Table 3 - Approximately Here]

[Table 4 - Approximately Here]

Analysis of the data, using the method shown in Section 2.3, results in response surface models for the mean and variance of each response as follows:

$\hat{\mu_{1 S}}(\mathbf{x})=2.1725-0.1913 x_{1}-0.1470 x_{2}+0.0613 x_{1}^{2}-0.1163 x_{2}^{2}-0.2375 x_{1} x_{2}$ 


$$
\begin{aligned}
& \hat{\sigma_{1 S}^{2}}(\mathbf{x})=0.03300-0.0004 x_{1}-0.0008 x_{2}-0.0154 x_{1}^{2}-0.0151 x_{2}^{2}+0.0013 x_{1} x_{2} \\
& \hat{\mu_{2 N}}(\mathbf{x})=10.0000+0.0497 x_{1}+0.0434 x_{2}-0.0381 x_{1}^{2}-0.0256 x_{2}^{2}-0.055 x_{1} x_{2} \\
& \hat{\sigma_{2 N}^{2}}(\mathbf{x})=0.0058+0.0001 x_{1}-0.0022 x_{2}+0.0011 x_{1}^{2}-0.0006 x_{2}^{2}+0.0013 x_{1} x_{2} \\
& \hat{\mu_{3 L}}(\mathbf{x})=94.9775+0.4832 x_{1}+0.7465 x_{2}-0.3725 x_{1}^{2}-0.3175 x_{2}^{2}+0.1550 x_{1} x_{2} \\
& \hat{\sigma_{3 L}^{2}}(\mathbf{x})=0.1898-0.0011 x_{1}-0.0039 x_{2}-0.0942 x_{1}^{2}-0.0902 x_{2}^{2}+0.0013 x_{1} x_{2}
\end{aligned}
$$

Using Equations (11) through (16), the proposed VPMRD optimization model is then applied to obtain the optimum operating conditions for the filtration process, as shown in Table 5. In this particular example, the constraints are comprised of the bounds on the responses that must be strictly adhered to. These bounds are denoted by the technical requirements of the system stated as the USL and/or LSL. Specifically, filtration time must be less than 7 seconds, filtration volume must be within the range of $9.5-10.5 \mathrm{~mL}$, and filtration purity must be greater than zero. Further, the goals are determined based on the desire to minimize the process bias and variance. For this example, the target values of interest are theoretically zero seconds for filtration time, $10 \mathrm{~mL}$ for filtration volume, and $100 \%$ for filtration purity. Additionally, we specify that the target for the variance is equal to zero because the goal of an RD study is always to minimize the variance. Given these constraints and their associated deviation variables, the deviation function to be minimized considers the over-achievement for the $S$ - and $N$-type quality characteristics and the under-achievement for the $N$ - and $L$-type quality characteristics of interests for the mean and the over-achievement of each quality characteristic for the variance. Here, the streamlined procedure for preemptive goal programming is used to first minimize the variance and then attempt to achieve the mean equal to the desired target value where all 
responses of interest are weighted equally. The big $M$ method [3] is utilized to establish a prioritization scheme within the optimization process. Here, the deviation variables associated with the variance are given substantially larger weights, designated by $\mathrm{M}$, over that of the deviation variables associated with the process bias.

\section{[Table 5 - Approximately Here]}

The results of the proposed method, which are shown in Table 6, indicate the goal of zero variance for filtration purity was achieved; yet, the variance of both filtration time and volume slightly exceed zero. In addition, the mean of each response is achieved with minimal amounts of bias allowed; however, none of the mean values achieved the exact target value desired.

[Table 7 - Approximately Here]

\section{Comparison study}

In this section, we validate the proposed methodology by comparing its results to those obtained using traditional RD optimization models, including both the dual response approach (Vining and Myers, 1990) and the MSE model (Lin and Tu, 1995). The dual response approach minimizes the variance with the constraint that the process mean equals the desired target value, which can be written as

$$
\begin{aligned}
& \min \hat{\sigma}^{2}(\mathbf{x}) \\
& \text { s.t. } \hat{\mu}(\mathbf{x})=\tau \\
& \mathbf{x} \in \Omega
\end{aligned}
$$

where $\Omega$ is the region of interest. This optimization model is typically used for problems in which it is critical that the quality characteristic of interest adheres strictly to the desired target 
value. Given this approach, we obtain optimum operating conditions that result in the mean located on target with some amount of variation around the mean.

To improve upon the dual response approach, Lin and $\mathrm{Tu}$ (1995) relaxed its zero-bias assumption (i.e. the constraint requiring that the process mean must equal the desired target value) and proposed a model that simultaneously minimizes the squared difference of the mean from the target value and the variance as follows:

$$
\begin{aligned}
& \min (\hat{\mu}(\mathbf{x})-\tau)^{2}+\hat{\sigma}^{2}(\mathbf{x}) \\
& \text { s.t. } \mathbf{x} \in \Omega
\end{aligned}
$$

When minimizing the variability of the response of interest is of equal or greater importance than achieving the desired target value, this optimization strategy is often utilized. Based on such an approach, it is observed that by allowing some difference between the mean and the desired target value, the resulting process variance is less than or at most equal to the variance of the dual response approach.

For the purpose of comparison, we utilize an approach similar to that demonstrated by Tang and $\mathrm{Xu}$ (1995) in which we approximate the traditional RD models using the VPMRD framework developed in this paper. Here, we reformulate the original RD models as goal programming problems and apply them to the example used previously to illustrate our proposed methodology. The formulations of and the results obtained from these equivalent models are presented in the following sections. The results obtained using these models are then compared to the results obtained from our proposed methodology.

Expansion of the dual response approach for multiresponse problems 
Using the dual response approach, the first priority of the optimization procedure is to achieve the desired target value and then the model attempts to minimize the variance. To approximate the optimization procedure of the dual response approach for a multiresponse problem, we use a preemptive goal programming approach, which is similar to that used in the proposed model. As discussed previously, all quality characteristics are equally weighted; yet in this case, substantially larger weighted priorities are placed on the deviation variables associated with minimizing the process bias over that of the deviation variables associated with minimizing the variance. Given the same response surface models and constraints used to demonstrate the proposed model, the big $\mathrm{M}$ method can be used in the optimization procedure to establish a prioritization scheme that reflects the dual response approach for multiple quality characteristics as follows:

$$
Z=\left[(1 / 3)\left(d_{2}^{+}\right)+(1 / 3)\left(d_{4}^{+}\right)+(1 / 3)\left(d_{6}^{-}\right)+M\left(d_{1}^{+}\right)+M\left(d_{3}^{-}+d_{3}^{+}\right)+M\left(d_{5}^{-}\right)\right]
$$

Based on Equation (19), we can interpret the objective of this model as an attempt to locate the mean at the desired target first and then an attempt is made to minimize the variance. Therefore, this equivalent model replicates the goals of the original dual response approach and allows us to optimize multiple quality characteristics simultaneously. The results obtained using this model are shown in Table 7.

[Table 7 Approximately Here]

\section{Expansion of the MSE model for multiresponse problems}

In terms of the MSE model, minimizing the squared difference of the mean from the desired target value and minimizing the variance are of equal priority in the optimization procedure and are considered simultaneously. The optimization procedure of the MSE model can be 
approximated for multiresponse problems using a nonpreemptive goal programming approach (Hillier and Liberman, 2001). To create this equivalent model, equal weights are given to the variables in the deviation function for the mean and variance. Again, based on the response surface models and constraints used to illustrate the proposed model, we establish a prioritization scheme that reflects the MSE model for multiresponse problems, which can be written as

$$
\min Z=\left[\left(d_{2}^{+}\right)+\left(d_{4}^{+}\right)+\left(d_{6}^{-}\right)+\left(d_{1}^{+}\right)+\left(d_{3}^{-}+d_{3}^{+}\right)+\left(d_{5}^{-}\right)\right]
$$

From Equation (20), we can observe that minimizing the bias and minimizing the variance are of equal importance. This prioritization scheme effectively replicates the original intention of the MSE model, and this formulation also allows us to optimize multiple quality characteristics simultaneously. The results obtained using this model are shown in Table 8.

[Table 8 Approximately Here]

\section{Discussion of comparison study results}

The results of this study comparing the proposed approach to that of the prioritization schemes associated with traditional RD optimization models are best considered based on the evaluation of the mean and variance at their optimal settings. In terms of the variance, Table 9 shows the optimum operating conditions for each approach, which also represents the deviation from the target, given that the desired target value is zero. The only result that indicates the goal of zero variance was achieved is for the response of filtration purity using the proposed VPMRD approach; all other results slightly exceed zero. Yet, it is important to note that the proposed method also resulted in the lowest variance for the response of filtration time in comparison to the other approaches. In terms of the mean, Table 10 shows the evaluation of the mean at the optimum operating conditions and the deviations of the mean from the target value for each 
optimization method considered here. For filtration time, all optimization methods over-achieve the target, with the multiresponse expansion of the dual response concept producing the minimum deviation. In terms of filtration volume and purity, all approaches under-achieve the target. Here, the multiresponse expansion of the MSE and the dual response concepts produce the minimum deviation for the filtration volume and purity, respectively.

[Table 9 - Approximately Here]

[Table 10 - Approximately Here]

As has been shown with other RD models in the past, we see here that a trade-off exists between minimizing the variance and minimizing the process bias. Based on the results of this comparison study, it can be concluded that the proposed model achieves its primary goal of first minimizing the variance and then attempting to achieve the mean equal to the desired target value. This logic explains why this method tended to produce the minimum variance, but not the minimum process bias. Yet, this approach provides a flexible and structured method for modeling multiresponse RD problems in the presence of responses with differing objectives. Therefore, this method is useful in situations where minimizing the variance is more critical than achieving a specific target value for problems involving multiple objectives.

\section{Conclusion}

In this work, a new RD optimization approach and framework was proposed, called VPMRD, to handle situations in which we wish to determine the optimum operating conditions for a system when the problem entails multiple responses and in cases where it is critically important that the variance of the responses being considered is minimized. Here, the proposed method utilized goal programming techniques to model a multiresponse problem in a single objective function 
using deviation variables. The objective of the proposed model addressed the inherent goal of $\mathrm{RD}$ where the first priority was to minimize the variance. The proposed optimization approach was described in detail and illustrated though the use of a numerical example. The results obtained from this approach were then compared to that of expanded approaches of the traditional RD optimization models in order to address multiple quality characteristics.

The result of this work provides a method for using RD in real-world situations where optimal solutions are desired in the face of multiple responses. The approach discussed here illustrates that there are trade-offs in design between minimizing the variance and achieving the desired target value. Yet, the specific model proposed here addresses these trade-offs by providing a flexible and structured method for modeling multiresponse RD problems. 


\section{References}

Cho BR, Park C. Robust design modeling and optimization with unbalanced data. Computers and Industrial Engineering 2005;48(2): 173-80.

Hillier FS, Lieberman GJ. Introduction to operations research. New York: McGraw-Hill, 2001.

Kim YJ, Cho BR. Development of priority-based robust design. Quality Engineering 2002;14(3): 355-63.

Kovach J, Cho BR. A D-optimal design approach to robust design under constraints: a new design for six sigma tool. International Journal of Six Sigma and Competitive Advantage 2006;2(4): 389-403.

Lee SB, Park C, Cho BR. Development of a highly efficient and resistant robust design. International Journal of Production Research 2007;45(1): 157-67.

Lin DKJ, Tu W. Dual response surface optimization. Journal of Quality Technology 1995;27(1): 34-9.

Myers RH, Carter WH. Response surface techniques for dual response systems. Technometrics 1973;15(2): 301-17.

Nair VN, Shoemaker AC, The role of experimentation in quality engineering: a review of Taguchi's contributions. In: Ghosh, S (Ed.). Statistical design and analysis of industrial experiments. New York: Marcel Dekker, 1990. p. 247-77.

Park C, Cho BR. Development of robust design under contaminated and non-normal data. Quality Engineering 2003;15(3): 463-69.

Shin S, Cho BR. Bias-specified robust design optimization and its analytical solutions. Computers and Industrial Engineering 2005;48(1): 129-40.

Taguchi G. Introduction to quality engineering: designing quality into products and processes. New York: Krauss International Publications, 1986.

Taguchi G. System of experimental design: engineering methods to optimize quality and minimize costs. Dearborn, MI: American Supplier Institute, 1987.

Tang LC, Xu K. A unified approach for dual response surface optimization. Journal of Quality Technology 2002;34(4): 437-47.

Vining GC, Myers RH. Combining Taguchi and response surface philosophies - a dual response approach. Journal of Quality Technology 1990;22(1): 38-45. 
Figures and Tables

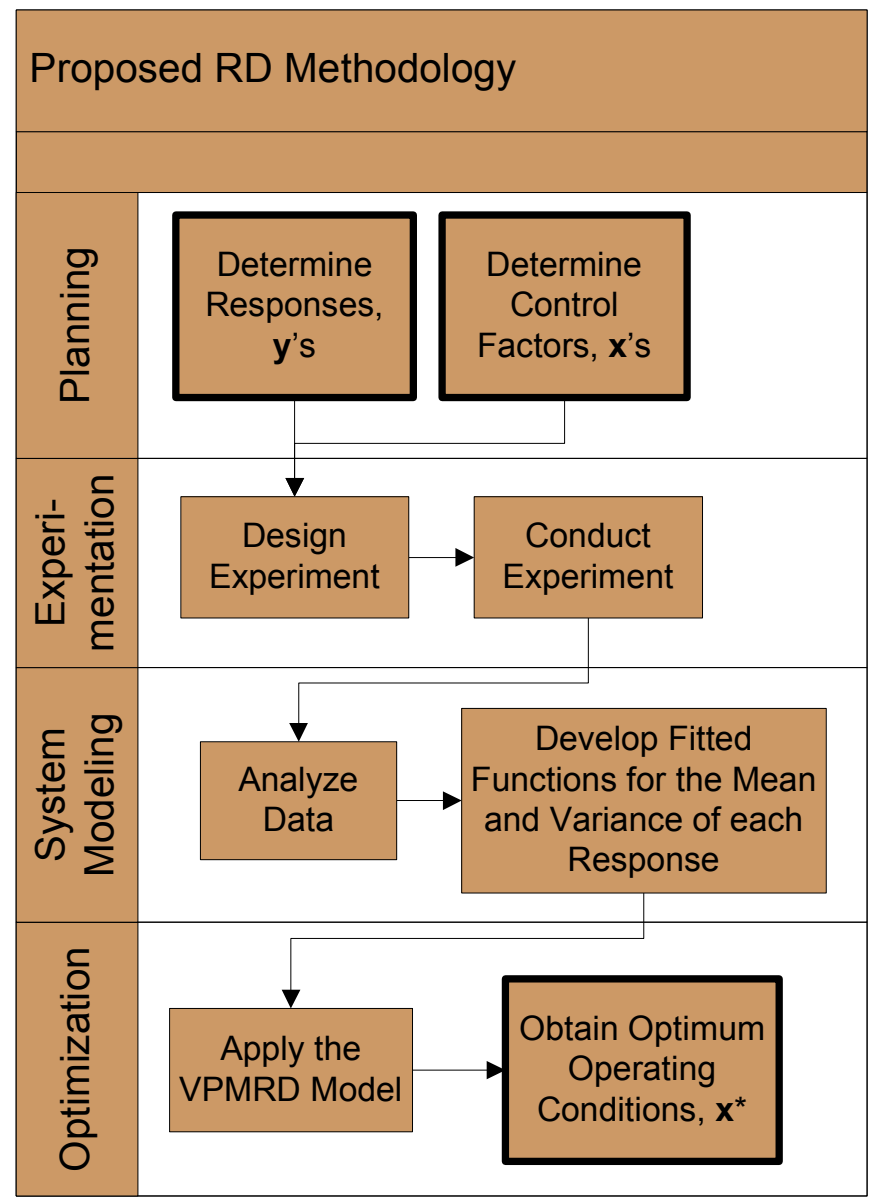

Figure 1 A Process Map of the Proposed Methodology 
Table 1 General Model of the Proposed VPRMD Framework

\begin{tabular}{|l|l|}
\hline Given & $\begin{array}{l}\text { Design factors: } x_{i}, i=1,2, \ldots, n \\
\text { Responses: } y_{k t}, \text { where } \\
\quad k=1,2, \ldots, a, \ldots, b, \ldots, c \\
\quad t=\text { type of quality characteristic }(S-, N-, \text { or } L-)\end{array}$ \\
& $\begin{array}{l}\text { Fitted response models: Mean: } \hat{\mu_{k t}}(\mathbf{x}) \text { and variance: } \hat{\sigma}_{k t}^{2}(\mathbf{x}) \\
\text { Desired target values: Mean: } \tau_{\hat{\mu_{k t}}} \text { and variance: } \tau_{\hat{\sigma_{k t}^{2}}}^{2}\end{array}$ \\
\hline Find & $\begin{array}{l}\text { Robust design factor specifications: } x_{i}^{*}, i=1,2, \ldots, n \\
\text { Deviation variables associated with constraints: } d_{g}^{-}, d_{g}^{+}, g=1,2, \ldots, r\end{array}$ \\
\hline Satisfy & $\begin{array}{l}\text { Constraints: } \\
1 . \quad \text { System requirements } \\
\text { 2. System goals }\end{array}$ \\
\hline Minimize & $Z=\left[f_{1}\left(d_{1}^{-}, d_{1}^{+}\right), f_{2}\left(d_{2}^{-}, d_{2}^{+}\right), \ldots, f_{r}\left(d_{r}^{-}, d_{r}^{+}\right)\right]$ \\
\hline
\end{tabular}

Table 2 Experimental Design and Observations of Filtration Time for the Multiresponse Chemical Filtration Study

\begin{tabular}{|c|r|r|r|c|c|c|c|}
\cline { 2 - 6 } \multicolumn{1}{c|}{} & Temperature & Pressure & \multicolumn{3}{c|}{$\begin{array}{c}\text { Filtration Time } \\
\text { (seconds) }\end{array}$} & \multirow{2}{*}{ Average } & \multirow{2}{*}{ Variance } \\
\cline { 2 - 6 } \multicolumn{1}{c|}{ Coded Units } & \multicolumn{3}{c|}{ 3 Replications } & & \\
\hline Treatment No. & $x_{1}$ & $x_{2}$ & $y_{1 S 1}$ & $y_{1 S 2}$ & $y_{1 S 3}$ & $\bar{y}_{1 S}$ & $s_{1 S}^{2}$ \\
\hline 1 & -1 & -1 & 3.86 & 4.03 & 3.92 & 3.94 & 0.007 \\
\hline 2 & 1 & -1 & 3.12 & 3.07 & 3.02 & 3.07 & 0.003 \\
\hline 3 & -1 & 1 & 2.82 & 2.79 & 2.87 & 2.83 & 0.002 \\
\hline 4 & 1 & 1 & 1.07 & 0.97 & 0.99 & 1.01 & 0.003 \\
\hline 5 & -1.414 & 0 & 1.30 & 1.26 & 1.32 & 1.29 & 0.001 \\
\hline 6 & 1.414 & 0 & 2.07 & 2.14 & 2.11 & 2.11 & 0.001 \\
\hline 7 & 0 & -1.414 & 0.60 & 0.63 & 0.68 & 0.64 & 0.002 \\
\hline 8 & 0 & 1.414 & 2.03 & 2.08 & 2.04 & 2.05 & 0.001 \\
\hline 9 & 0 & 0 & 2.12 & 1.79 & 2.16 & 2.02 & 0.041 \\
\hline 10 & 0 & 0 & 2.80 & 2.52 & 2.42 & 2.58 & 0.039 \\
\hline 11 & 0 & 0 & 2.19 & 2.02 & 2.14 & 2.12 & 0.008 \\
\hline 12 & 0 & 0 & 1.96 & 1.77 & 2.19 & 1.97 & 0.044 \\
\hline
\end{tabular}


Table 3 Experimental Design and Observations of Filtration Volume for the Multiresponse Chemical Filtration Study

\begin{tabular}{|c|r|r|c|c|c|c|c|}
\cline { 2 - 6 } \multicolumn{1}{c|}{} & Temperature & Pressure & \multicolumn{3}{c|}{ Filtration Volume $(\mathrm{mL})$} & \multirow{2}{*}{ Average } & \multirow{2}{*}{ Variance } \\
\cline { 2 - 6 } \multicolumn{1}{c|}{ Coded Units } & \multicolumn{3}{c|}{ 3 Replications } & & \\
\hline Treatment No. & $x_{1}$ & $x_{2}$ & $y_{2 N 1}$ & $y_{2 N 2}$ & $y_{2 N 3}$ & $\bar{y}_{2 N}$ & $s_{2 N}^{2}$ \\
\hline 1 & -1 & -1 & 9.70 & 9.79 & 9.73 & 9.74 & 0.002 \\
\hline 2 & 1 & -1 & 9.96 & 9.95 & 9.93 & 9.95 & 0.000 \\
\hline 3 & -1 & 1 & 9.94 & 9.96 & 9.97 & 9.96 & 0.000 \\
\hline 4 & 1 & 1 & 10.00 & 9.97 & 9.89 & 9.95 & 0.003 \\
\hline 5 & -1.414 & 0 & 9.78 & 9.87 & 10.01 & 9.89 & 0.013 \\
\hline 6 & 1.414 & 0 & 10.02 & 10.15 & 9.92 & 10.03 & 0.013 \\
\hline 7 & 0 & -1.414 & 9.80 & 10.04 & 9.98 & 9.94 & 0.016 \\
\hline 8 & 0 & 1.414 & 10.10 & 9.99 & 10.01 & 10.03 & 0.003 \\
\hline 9 & 0 & 0 & 10.12 & 10.01 & 9.86 & 10.00 & 0.001 \\
\hline 10 & 0 & 0 & 10.10 & 9.97 & 9.85 & 9.97 & 0.001 \\
\hline 11 & 0 & 0 & 10.08 & 9.99 & 10.13 & 10.07 & 0.017 \\
\hline 12 & 0 & 0 & 9.98 & 10.11 & 9.78 & 9.96 & 0.004 \\
\hline
\end{tabular}

Table 4 Experimental Design and Observations of Filtration Purity for the Multiresponse Chemical Filtration Study

\begin{tabular}{|c|r|r|c|c|c|c|c|}
\cline { 2 - 7 } \multicolumn{1}{c|}{} & Temperature & Pressure & \multicolumn{3}{c|}{ Filtration Purity $(\%)$} & \multirow{2}{*}{ Average } & \multirow{2}{*}{ Variance } \\
\cline { 2 - 7 } \multicolumn{1}{c|}{ Coded Units } & \multicolumn{3}{c|}{ 3 Replications } & & \\
\hline Treatment No. & $x_{1}$ & $x_{2}$ & $y_{3 L 1}$ & $y_{3 L 2}$ & $y_{3 L 3}$ & $\bar{y}_{3 L}$ & $s_{3 L}^{2}$ \\
\hline 1 & -1 & -1 & 93.09 & 92.99 & 93.03 & 93.04 & 0.003 \\
\hline 2 & 1 & -1 & 93.76 & 93.83 & 93.81 & 93.80 & 0.001 \\
\hline 3 & -1 & 1 & 94.33 & 94.35 & 94.3 & 94.33 & 0.001 \\
\hline 4 & 1 & 1 & 95.64 & 95.76 & 95.72 & 95.71 & 0.004 \\
\hline 9 & -1.414 & 0 & 93.59 & 93.73 & 93.76 & 93.69 & 0.008 \\
\hline 10 & 1.414 & 0 & 94.94 & 94.88 & 94.9 & 94.91 & 0.001 \\
\hline 11 & 0 & -1.414 & 93.41 & 93.28 & 93.59 & 93.43 & 0.024 \\
\hline 12 & 0 & 1.414 & 95.39 & 95.42 & 95.36 & 95.39 & 0.001 \\
\hline 13 & 0 & 0 & 94.37 & 95.17 & 94.64 & 94.73 & 0.166 \\
\hline 14 & 0 & 0 & 95.36 & 95.63 & 94.99 & 95.33 & 0.103 \\
\hline 15 & 0 & 0 & 95.76 & 94.93 & 95.43 & 95.37 & 0.175 \\
\hline 16 & 0 & 0 & 94.12 & 94.2 & 95.13 & 94.48 & 0.315 \\
\hline
\end{tabular}


Table 5 Proposed VPMRD Optimization Model

for the Multiresponse Chemical Filtration Study

\begin{tabular}{|c|c|}
\hline Given & 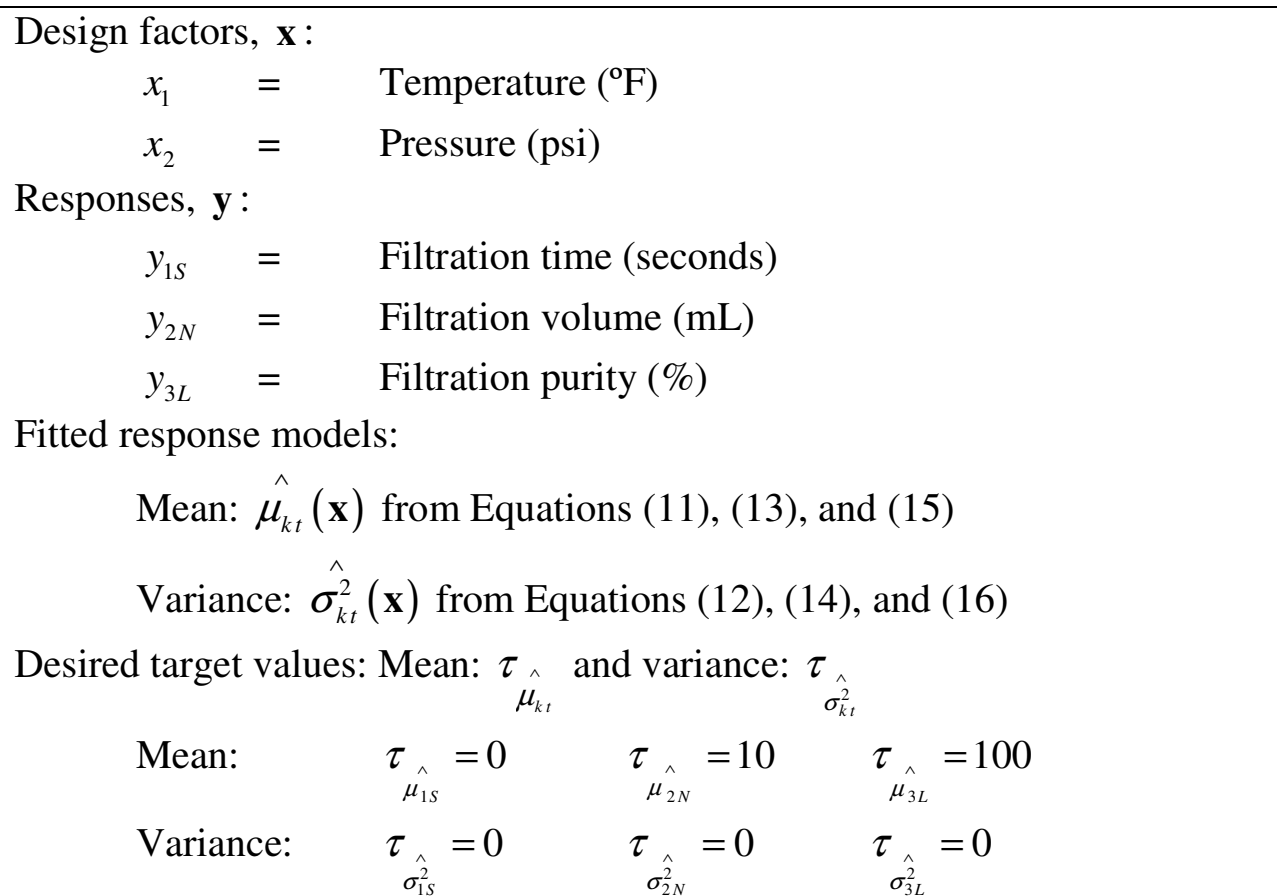 \\
\hline Find & $\begin{array}{l}\text { Robust design factor specifications: } x_{i}^{*}, i=1,2 \\
\text { Deviation variables associated with goals: } d_{g}^{-}, d_{g}^{+}, g=1,2, \ldots, 6\end{array}$ \\
\hline Satisfy & 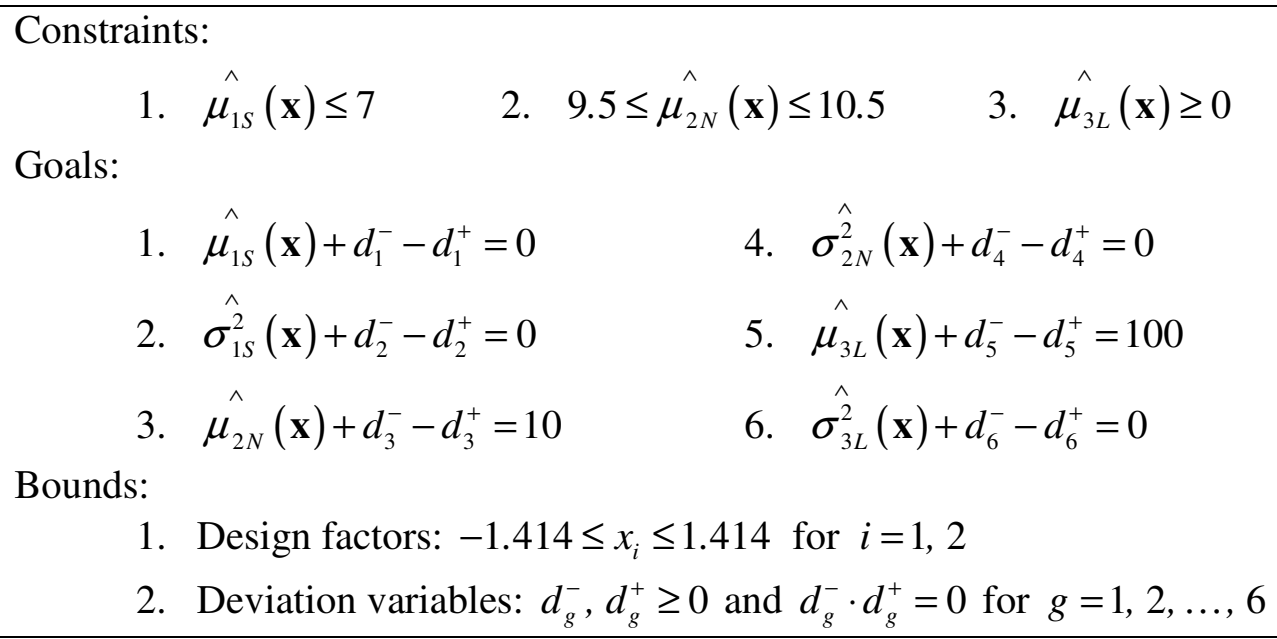 \\
\hline Minimize & $Z=\left[M\left(d_{2}^{+}\right)+M\left(d_{4}^{+}\right)+M\left(d_{6}^{+}\right)+(1 / 3)\left(d_{1}^{+}\right)+(1 / 3)\left(d_{3}^{-}+d_{3}^{+}\right)+(1 / 3)\left(d_{5}^{-}\right)\right]$ \\
\hline
\end{tabular}


Table 6 Results of the Proposed VPMRD Approach for the Multiresponse Chemical Filtration Study

\begin{tabular}{|c|lll|}
\hline Optimal Settings & $\mathbf{x}^{*}=$ & $(1.4117,0.0651)$ \\
\hline \multirow{4}{*}{ Mean } & $\hat{\mu_{1 S}}(\mathbf{x})$ & $=$ & 1.9927 \\
& $\hat{\mu_{2 N}}(\mathbf{x})$ & $=$ & 9.9919 \\
& $\hat{\mu_{3 L}}(\mathbf{x})$ & $=$ & 94.9788 \\
\hline \multirow{4}{*}{ Variance } & $\hat{\sigma_{1 S}^{2}}(\mathbf{x})$ & $=$ & 0.0017 \\
& $\hat{\sigma_{2 N}^{2}}(\mathbf{x})$ & $=$ & 0.0081 \\
& $\hat{\sigma_{3 L}^{2}}(\mathbf{x})$ & $=$ & 0 \\
\hline
\end{tabular}

Table 7 Results of the Multiresponse Expansion of the Dual Response Approach for the Multiresponse Chemical Filtration Study

\begin{tabular}{|l|lll|}
\hline Optimal Settings & $\mathbf{x}^{*}=$ & $(0.5444,1.2825)$ \\
\hline \multirow{4}{*}{ Mean } & $\hat{\mu_{1 S}}(\mathbf{x})$ & $=$ & 1.5409 \\
& $\hat{\mu_{2 N}}(\mathbf{x})$ & $=$ & 9.9909 \\
& $\hat{\mu_{3 L}}(\mathbf{x})$ & $=$ & 95.6735 \\
\hline \multirow{4}{*}{ Variance } & $\hat{\sigma_{1 S}^{2}}(\mathbf{x})$ & $=$ & 0.0033 \\
& $\hat{\sigma_{2 N}^{2}}(\mathbf{x})$ & $=$ & 0.0033 \\
& $\hat{\sigma_{3 L}^{2}}(\mathbf{x})$ & $=$ & 0.0088 \\
\hline
\end{tabular}


Table 8 Results of the Multiresponse Expansion of the MSE Model for the Multiresponse Chemical Filtration Study

\begin{tabular}{|c|lll|}
\hline Optimal Settings & $\mathbf{x}^{*}=$ & $(0.4759,1.2135)$ \\
\hline \multirow{4}{*}{ Mean } & $\hat{\mu_{1 S}}(\mathbf{x})$ & $=$ & 1.6086 \\
& $\hat{\mu_{2 N}}(\mathbf{x})$ & $=$ & 9.9982 \\
& $\hat{\mu_{3 L}}(\mathbf{x})$ & $=$ & 95.6509 \\
\hline \multirow{4}{*}{ Variance } & $\hat{\sigma_{1 S}^{2}}(\mathbf{x})$ & $=$ & 0.0069 \\
& $\hat{\sigma_{2 N}^{2}}(\mathbf{x})$ & $=$ & 0.0033 \\
& $\hat{\sigma_{3 L}^{2}}(\mathbf{x})$ & $=$ & 0.0311 \\
\hline
\end{tabular}

Table 9 Comparison of Optimization Methods in Determining the Optimal Design Factor Settings and the Evaluation of the Variance for the Multiresponse Chemical Filtration Study

\begin{tabular}{|l|c|c|c|c|}
\hline \multicolumn{1}{|c|}{ Method } & $\begin{array}{c}\text { Optimal Settings } \\
\left(x_{1}^{*}, x_{2}{ }^{*}\right)\end{array}$ & $\hat{\sigma_{1 S}^{2}}(\mathbf{x})$ & $\hat{\sigma_{2 N}^{2}}(\mathbf{x})$ & $\hat{\sigma_{3 L}^{2}}(\mathbf{x})$ \\
\hline $\begin{array}{l}\text { Multiresponse } \\
\text { Expansion of the } \\
\text { Dual Response } \\
\text { Approach }\end{array}$ & $(0.5444,1.2825)$ & 0.0033 & 0.0033 & 0.0088 \\
\hline $\begin{array}{l}\text { Multiresponse } \\
\text { Expansion of the } \\
\text { MSE Model }\end{array}$ & $(0.4759,1.2135)$ & 0.0069 & 0.0033 & 0.0311 \\
\hline $\begin{array}{l}\text { Proposed VPMRD } \\
\text { Approach }\end{array}$ & $(1.4117,0.0651)$ & 0.0017 & 0.0081 & 0 \\
\hline
\end{tabular}


Table 10 Comparison of Optimization Methods in Determining the Optimal Design Factor Settings and the Evaluation of the Mean for the Multiresponse Chemical Filtration Study

\begin{tabular}{|l|c|c|c|c|c|c|c|}
\hline \multicolumn{1}{|c|}{ Method } & $\begin{array}{c}\text { Optimal Settings } \\
\left(x_{1}{ }^{*}, x_{2}{ }^{*}\right)\end{array}$ & $\hat{\mu_{1 S}(\mathbf{x})}$ & $d_{1}^{+}$ & $\hat{\mu_{2 N}(\mathbf{x})}$ & $d_{3}^{-}$ & $\hat{\mu_{3 L}}(\mathbf{x})$ & $d_{5}^{-}$ \\
\hline $\begin{array}{l}\text { Multiresponse } \\
\text { Expansion of } \\
\text { the Dual } \\
\begin{array}{l}\text { Response } \\
\text { Approach }\end{array}\end{array}$ & $(0.5444,1.2825)$ & 1.5409 & 1.5409 & 9.9909 & 0.0091 & 95.6735 & 4.3265 \\
\hline $\begin{array}{l}\text { Multiresponse } \\
\text { Expansion of } \\
\text { the MSE } \\
\text { Model }\end{array}$ & $(0.4759,1.2135)$ & 1.6086 & 1.6086 & 9.9982 & 0.0018 & 95.6509 & 4.3491 \\
\hline $\begin{array}{l}\text { Proposed } \\
\text { VPMRD } \\
\text { Approach }\end{array}$ & $(1.4117,0.0651)$ & 1.9927 & 1.9927 & 9.9919 & 0.0081 & 94.9788 & 5.0212 \\
\hline
\end{tabular}

